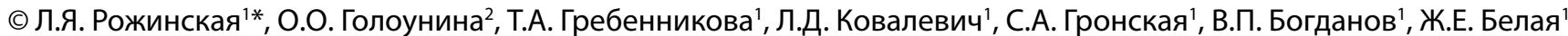

'ФГБУ «Национальный медицинский исследовательский центр эндокринологии» Минздрава России, Москва, Россия ФГАОУ ВО «Первый Московский государственный медицинский университет им. И.М. Сеченова» Минздрава России (Сеченовский Университет), Москва, Россия

Первичные гипероксалурии представляют собой группу редких генетических заболеваний, обусловленных дефицитом специфических печеночных ферментов обмена глиоксиловой кислоты, приводящих к гиперпродукции оксалатов с последующим их отложением в различных органах и тканях. Клинически заболевание проявляется рецидивирующим нефролитиазом и нефрокальцинозом, быстро прогрессирующей хронической болезнью почек (ХБП), впоследствии приводящей к терминальной стадии хронической почечной недостаточности, системному оксалозу, ПТГ-независимой гиперкальциемии, панцитопении, оксалатной остеопатии с остеосклерозом, патологическим переломам, эндокринопатиям. Независимо от типа первичной гипероксалурии, любая консервативная терапия является паллиативной и позволяет лишь замедлить прогрессирование заболевания, но не предотвратить его полностью. В статье представлено первое описание клинического случая лечения молодого пациента с генетически подтвержденным врожденным нарушением обмена оксалатов, перенесшего трансплантации печени и почки через 10 лет от начала заболевания в период терминальной стадии ХБП, препаратом моноклонального человеческого антитела к лиганду рецептора активатора ядерного фактора каппа бета (RANKL) - деносумабом - с положительным клиническим эффектом. Знание особенностей клинических проявлений, своевременная диагностика и лечение первичной гипероксалурии имеют важное прогностическое значение для пациентов.

КЛЮЧЕВЫЕ СЛОВА: гипероксалурия; мочекаменная болезнь; хроническая болезнь почек; остеосклероз; гиперкальциемия; деносумаб; клинический случай

\section{CLINICAL EXPERIENCE OF USING DENOSUMAB IN THE TREATMENT OF HYPERCALCEMIA AND OXALATE OSTEOPATHY FOR A YOUNG PATIENT WITH PRIMARY HYPEROXALURIA TYPE 1}

(c) Liudmila Ya. Rozhinskaya1* , Olga O. Golounina², Tatiana A. Grebennikova1', Liliya D. Kovalevich1', Sofya A. Gronskaya', Victor P. Bogdanov' ${ }^{1}$ Zhanna E. Belaya'

'The National Medical Research Center for Endocrinology of the Ministry of Health of the Russian Federation, Moscow, Russia

${ }^{2}$ I.M. Sechenov First Moscow State Medical University of the Ministry of Health of the Russian Federation (Sechenov University), Moscow, Russia

Primary hyperoxaluria are a group of rare genetic diseases caused by defective glyoxylate metabolism leading to excessive oxalate production and deposition into the tissues (oxalosis). Clinical manifestations include recurrent nephrolithiasis and nephrocalcinosis, rapidly progressive chronic kidney disease subsequently leading to end-stage renal disease, systemic oxalosis, PTH-independent hypercalcemia, pancytopenia, oxalate osteopathy with osteosclerosis, pathological fractures and endocrinopathy. Regardless of the type of primary hyperoxaluria any conservative therapy is palliative and can only slow the progression of the disease but not prevent it completely. We report the case of a young male patient with genetically confirmed primary hyperoxaluria type 1 complicated by severe oxalate osteopathy and hypercalcemia, who received a combined liver/ kidney transplant after 10 years from the disease. Treatment with human monoclonal antibody to the receptor activator of nuclear factor kappa B ligand (RANKL) — denosumab allowed achieving normalization of calcium-phosphorus metabolism, significantly reduce the activity of bone resorption and improve clinical performance. Knowledge of the features of clinical manifestations, timely diagnosis and treatment of primary hyperoxaluria are important prognostic value for patients.

KEYWORDS: hyperoxaluria; urolithiasis disease; chronic kidney disease; osteosclerosis; hypercalcemia; denosumab; clinical case 


\section{АКТУАЛЬНОСТЬ}

Первичные гипероксалурии - это группа редких аутосомно-рецессивных заболеваний, обусловленных дефицитом специфических печеночных ферментов обмена глиоксиловой кислоты в результате мутаций в генах $A G X T$, GRHPR или HOGA1, приводящих к гиперпродукции оксалатов с последующим их отложением в различных органах и тканях [1]. Каждый фермент, кодируемый перечисленными генами, участвует в разных этапах метаболического пути глиоксилата. Это обусловливает клиническую гетерогенность заболевания и, следовательно, различные подходы к лечению первичных гипероксалурий. В зависимости от того, в каком гене произошла мутация, выделяют три типа первичной гипероксалурии, наиболее распространенным и тяжелым из которых является тип 1.

Первичная гипероксалурия типа 1 (ОМIM \# 259900) обусловлена мутацией в гене $A G X T$, кодирующем образование витамин В -зависимого фермента аланин-глиоксилатаминотрансферазы в пероксисомах клеток печени, основная функция которого - преобразование глиоксиловой кислоты в глицин. Генетически обусловленное нарушение метаболизма глиоксиловой кислоты приводит к усиленному накоплению в органеллах и цитоплазме гепатоцитов глиоксилата с последующим превращением в оксалат с образованием нерастворимых солей кальция $[2,3]$. Мутация c.508G >A (p.Gly170Arg) в гене $A G X T$, выявляемая в 25-40\% случаев, наиболее часто является причиной первичной гипероксалурии типа 1 в европейских и северо-американских популяциях $[4,5]$.

Согласно оценочным данным, распространенность первичной гипероксалурии типа 1 составляет 1-3 случая на 1000000 населения, заболеваемость - 1:120 000 новорожденных [6]. Клинически заболевание проявляется рецидивирующим нефролитиазом и нефрокальцинозом, быстро прогрессирующей хронической болезнью почек (ХБП), требующей заместительной почечной терапии, и приводит к системному оксалозу, ПТГ-независимой гиперкальциемии, панцитопении, оксалатной остеопатии с остеосклерозом, патологическим переломам, эндокринопатиям, в частности к гипогонадизму и гипотиреозу [7].

На сегодняшний день основным методом лечения первичной гипероксалурии типа 1 является комбинированная трансплантация печени и почки, позволяющая устранить дефект отсутствия фермента и восстановить нормальный обмен оксалатов $[8,9]$. Пациенты, перенесшие комбинированную трансплантацию, имеют лучшие показатели выживаемости по сравнению с пациентами, которым выполнена изолированная трансплантация почек [10]. По данным Научного регистра реципиентов трансплантации (Scientific Registry of Transplant Recipients, SRTR) и регистра Европейского общества детских нефрологов, Европейской ассоциации нефрологов и Европейской ассоциации по диализу и трансплантации (Registry of the European Society for Paediatric Nephrology/ European Renal Association - European Dialysis and Transplantation Association Registry, ESPN/ERA-EDTA Registry), 5-летняя выживаемость пациентов после комбинированной трансплантации печени и почек составляет 64-76\%, в то время как без трансплантации пятилетняя выживаемость после развития терминальной стадии ХБП не превышает 40\% [11, 12].
В 2020 г. Американским Агентством по контролю за пищевыми продуктами и медикаментами (FDA) был одобрен препарат Окслумо (Oxlumo) под торговым наименованием Лумасиран (Lumasiran ${ }^{\circledR}$ ) для лечения первичной гипероксалурии типа 1. Данный препарат снижает избыточную выработку оксалатов и, следовательно, концентрацию оксалатов в моче, однако применение Oxlumo в клинической практике в России пока недоступно.

При первичной гипероксалурии типа 2 (ОМІМ \# 260000) у больных присутствует дефицит D-глицератредуктазы и глиоксилатредуктазы - цитозольных ферментов, катализирующих превращение глиоксилата в гликолат и гидроксипирувата в D-глицерат в печени, обусловленный мутациями в гене GRHPR. Нарушение функции указанных ферментов приводит к повышенному образованию L-глицерата и оксалатов, однако последние образуются в меньшем количестве, чем при первичной гипероксалурии типа 1, поэтому первичная гипероксалурия типа 2 реже сопровождается развитием терминальной стадии ХБП [13]. Практически все мутации, описанные при первичной гипероксалурии типа 2, непосредственно влияют на кодирование или сплайсинг. Наиболее распространенной мутацией, выявляемой в 40\% случаев, является мутация c.103delG (p.Asp35Thrfs*11) в экзоне 2 гена GRHPR, приводящая к сдвигу рамки считывания с формированием преждевременного стоп-кодона $[14,15]$.

Первичная гипероксалурия типа 3 (ОМIM \# 613616) обусловлена мутациями в гене HOGA1, кодирующем белок, который преимущественно экспрессируется в митохондриях гепатоцитов и в почках. Этот митохондриальный фермент участвует в метаболическом пути гидроксипролина и катализирует конечную реакцию расщепление 4-гидрокси-2-оксоглутарата до пирувата и глиоксилата [16]. Мочекаменная болезнь, гематурия, почечная колика, мочевая инфекция обычно являются первыми проявлениями заболевания. Отличительная особенность пациентов с первичной гипероксалурией типа 3 - нормальная скорость клубочковой фильтрации (СКФ), отсутствие развития системного оксалоза и терминальной стадии ХБП [17].

Представляем клиническое наблюдение пациента с редким вариантом генетически опосредованного врожденного нарушения обмена оксалатов, осложненным тяжелой остеопатией и выраженной ПТГ-независимой гиперкальциемией.

\section{ОПИСАНИЕ СЛУЧАЯ}

Пациент А., 19 лет, обратился в отделение нейроэндокринологии и остеопатий ФГБУ «НМИЦ эндокринологии» Минздрава России с жалобами на сильные боли в костях таза, ограничение физической активности, общую и мышечную слабость, снижение массы тела.

Из анамнеза известно, что с детства страдал нефролитиазом, нефрокальцинозом. В связи с прогрессирующим ухудшением функции почек с возраста 11 лет находился на заместительной почечной терапии методом перитонеального диализа, с 17 лет переведен на гемодиализ. В возрасте 15 лет был обследован в лаборатории наследственных болезней обмена веществ Медико-генетического научного центра Москвы с целью исключения 
первичной гипероксалурии. Молекулярно-генетический анализ выявил мутацию с.508G>A в экзоне 4 гена AGXT в гетерозиготном состоянии, приводящую к замене Gly170Arg (СM910016), а также делецию двух нуклеотидов c.914 915delTG в экзоне 9 того же гена в гетерозиготном состоянии, что позволило установить диагноз «первичная гипероксалурия тип 1». В течение последующих двух лет пациент перенес удаление 15 зубов, а также экзостозов верхней и нижней челюсти.

В возрасте 18 лет проведена ортотопическая трансплантация печени с последующей трансплантацией почки от живого родственного донора. В послеоперационном периоде сохранялась удовлетворительная функция трансплантата, однако наблюдались признаки рецидивирующей инфекции мочевых путей, несмотря на постоянный прием уросептиков. Через год после трансплантации был госпитализирован с атакой пиелонефрита трансплантата, на фоне чего наблюдалось резкое снижение СКФ до 30 мл/мин/1,73 м². В том же году стали беспокоить боли в костях таза, купирующиеся приемом нестероидных противовоспалительных средств (НПВС). Тогда же, по данным лабораторных исследований, впервые была выявлена гиперкальциемия (кальций общий 3,62 ммоль/л) в сочетании с низким уровнем паратиреоидного гормона (ПТГ 3 нг/мл). По данным рентгенографии костей таза обращала на себя внимание высокая плотность костной ткани по типу остеопетроза.

В возрасте 20 лет пациент перенес острое нарушение мозгового кровообращения (ОНМК) по ишемическому типу в бассейне левой средней мозговой артерии с развитием легкого правостороннего центрального гемипареза.

При поступлении в отделение нейроэндокринологии и остеопатий пациент получал супрессивную терапию: такролимус пролонгированного действия 4,5 мг, метилпреднизолон 4 мг, микофенолата мофетил 250 мг 2 раза, а также севеламер 800 мг 3 раза в сутки, урсодезоксихолевую кислоту 250 мг 2 раза в день и разные дозы и виды $\mathrm{H} П \mathrm{BC}$.

При осмотре в отделении: рост 162 см, вес 43 кг, ИМТ $=16,4$ кг/м², АД 110/80 мм рт.Ст., ЧСС 76 уд/мин. Отмечались выраженная гипотрофия мышц, изменение осанки, сутулость, ограничение подвижности в левом тазобедренном суставе, болезненность костей при пальпации (рис. 1). При лабораторном обследовании выявлены панцитопения, гиперкальциемия, гиперфосфатемия в сочетании с низким уровнем ПТГ, повышение уровня гонадотропных гормонов (табл. 1, 2). При исследовании состояния костной системы обращало на себя внимание выраженное повышение маркеров костного ремоделирования: остеокальцин - 154,9 нг/мл (24-70), С-концевой телопептид коллагена I типа ( $\beta$-CrossLaps, CTX) 3,97 нг/мл $(0,1-0,85)$.

По результатам мультиспиральной компьютерной томографии (МСКТ) позвоночника и костей таза структура тел позвонков и костей таза с выраженными множественными участками остеосклероза, отмечается неравномерное утолщение кортикального слоя; в крестцово-подвздошных суставах субхондральные отделы неравномерно склерозированы, с участками разряжения костной ткани (рис. 2-4). По данным двухэнергетической рентгеновской абсорбциометрии (dual-energy X-ray

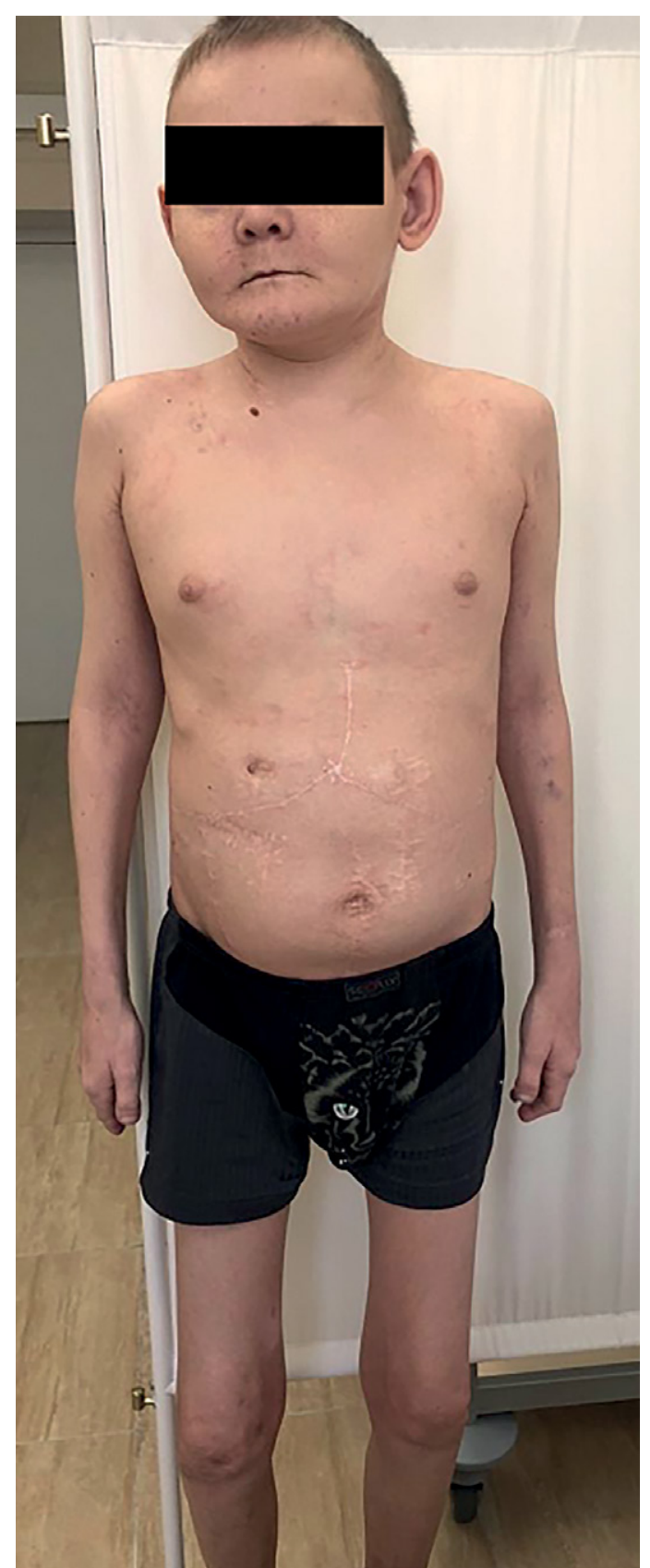

Рис. 1. Внешний вид пациента A.

absorptiometry, DXA) выявлено повышение минеральной плотности костной ткани во всех отделах скелета (табл. 3). По результатам ультразвукового исследования (УЗИ) органов мошонки - эхографические признаки кальцификатов обоих яичек, гипоплазии обоих яичек (размеры соответствуют 11-12 годам).

Учитывая сильные боли в костях, гиперкальциемию, высокие показатели костного метаболизма, была инициирована терапия деносумабом в дозе 60 мг подкожно (п/к). Поскольку препарат не зарегистрирован в Российской Федерации по данному показанию, пациент подписал информированное добровольное согласие на введение препарата off-label. Через 3 дня после инъекции деносумаба отмечена выраженная положительная динамика - снижение уровня общего кальция до 2,54 ммоль/л, фосфора до 0,99 ммоль/л, маркера костной резорбции $\beta$-CrossLaps до 0,63 нг/мл (см. табл. 1). Через 1 мес после п/к инъекции препарата 
Таблица 1. Данные лабораторного обследования пациента А. при первичном обращении в ФГБУ «НМИЦ эндокринологии» Минздрава России и в динамике

\begin{tabular}{|c|c|c|c|c|c|c|c|}
\hline \multirow[b]{2}{*}{ Параметр } & \multicolumn{6}{|c|}{ Значение } & \multirow[b]{2}{*}{$\begin{array}{c}\text { Референсный } \\
\text { интервал }\end{array}$} \\
\hline & 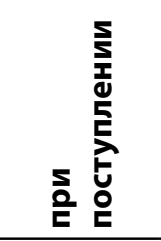 & 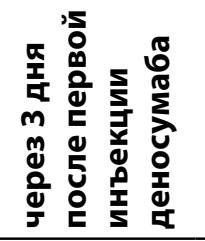 & 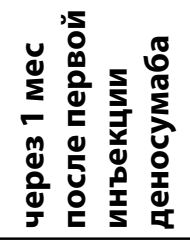 & 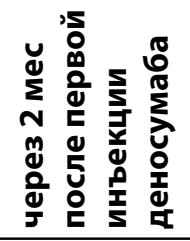 & 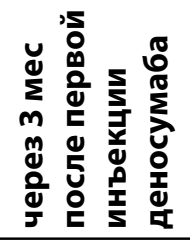 & 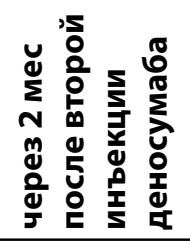 & \\
\hline Гемоглобин, г/л & 108 & - & 108 & 134 & 132 & 120 & $132-172$ \\
\hline Лейкоциты, кл/л & $2,91 \times 10^{9}$ & - & - & $4,1 \times 10^{9}$ & $4,0 \times 10^{9}$ & $3,65 \times 10^{9}$ & $3,9-10$ \\
\hline Тромбоциты, кл/л & $107 \times 10^{9}$ & - & $75 \times 10^{9}$ & $107 \times 10^{9}$ & $120 \times 10^{9}$ & $100 \times 10^{9}$ & $148-339$ \\
\hline $\begin{array}{l}\text { Кальций общий, } \\
\text { ммоль/л }\end{array}$ & 3,03 & 2,54 & 2,33 & 2,6 & 2,8 & 2,94 & $2,15-2,55$ \\
\hline $\begin{array}{l}\text { Кальций } \\
\text { ионизированный, } \\
\text { ммоль/л }\end{array}$ & 1,8 & - & - & 1,4 & - & - & $1,03-1,29$ \\
\hline Альбумин, г/л & 23 & 25 & 30,5 & 29 & 31 & 26 & $35-50$ \\
\hline $\begin{array}{l}\text { Кальций } \\
\text { с поправкой на } \\
\text { альбумин, ммоль/л }\end{array}$ & 3,43 & 2,82 & 2,52 & 2,79 & 2,98 & 3,22 & - \\
\hline Фосфор, ммоль/л & 1,69 & 0,99 & 1,02 & 1,76 & 1,87 & 1,58 & $0,74-1,52$ \\
\hline $\begin{array}{l}\text { Щелочная } \\
\text { фосфатаза, Ед/л }\end{array}$ & 187 & 138 & - & - & 190 & - & $40-150$ \\
\hline $\begin{array}{l}\text { Паратиреоидный } \\
\text { гормон (ПТГ), пг/мл }\end{array}$ & 8,12 & - & 9,5 & - & - & 5,94 & $15-65$ \\
\hline Остеокальцин, нг/мл & 154,9 & 235 & - & - & 148 & 121,7 & $24-70$ \\
\hline $\begin{array}{l}\text { С-концевой } \\
\text { телопептид } \\
\text { коллагена I типа, } \\
\text { нг/мл }\end{array}$ & 3,97 & 0,63 & - & - & 5,4 & 6 & $0,1-0,85$ \\
\hline Креатинин, мкмоль/л & 266,7 & 239,2 & 184 & 235 & 222 & 233 & $63-110$ \\
\hline $\begin{array}{l}\text { Скорость } \\
\text { клубочковой } \\
\text { фильтрации (СКФ), } \\
\text { мл/мин } / 1,73 \mathrm{M}^{2}\end{array}$ & 29 & 26 & 45 & 33 & 36 & 33 & $90-120$ \\
\hline АЛТ, Ед/л & 11 & - & 14,1 & - & 21 & 13 & $0-55$ \\
\hline АСТ, Ед/л & 46 & - & 41,3 & - & 56 & 45 & $5-34$ \\
\hline Калий, ммоль/л & 5,4 & 5,4 & 5,0 & - & 5,1 & 4,9 & $3,5-5,1$ \\
\hline Белок общий, г/л & 42 & - & 54,6 & - & 48,8 & 43 & 64-83 \\
\hline
\end{tabular}

Таблица 2. Данные гормонального обследования пациента А. при первичном обращении в ФГБУ «НМИЦ эндокринологии» Минздрава России

\section{Параметр}

Лютеинизирующий гормон (ЛГ), Ед/л

Фолликулостимулирующий гормон (ФСГ), Ед/л

Тестостерон, нмоль/л

Пролактин, мЕд/л

Тиреотропный гормон (ТTГ), MME/л

Т4 свободный, пмоль/л

\section{Значение}

11,2

19,4

14,4

405,3

4,654

11,97

\section{Референсный интервал}

$2,5-11$

$1,6-9,7$

$11-28,2$

$78-380$

$0,25-3,5$

9-19

Таблица 3. Остеоденситометрия пациента А.

\section{Область исследования}

LI-LIV
Neck
Total hip

\section{Z-критерий, SD}

$+4,2$
$+2,5$
$+5,0$



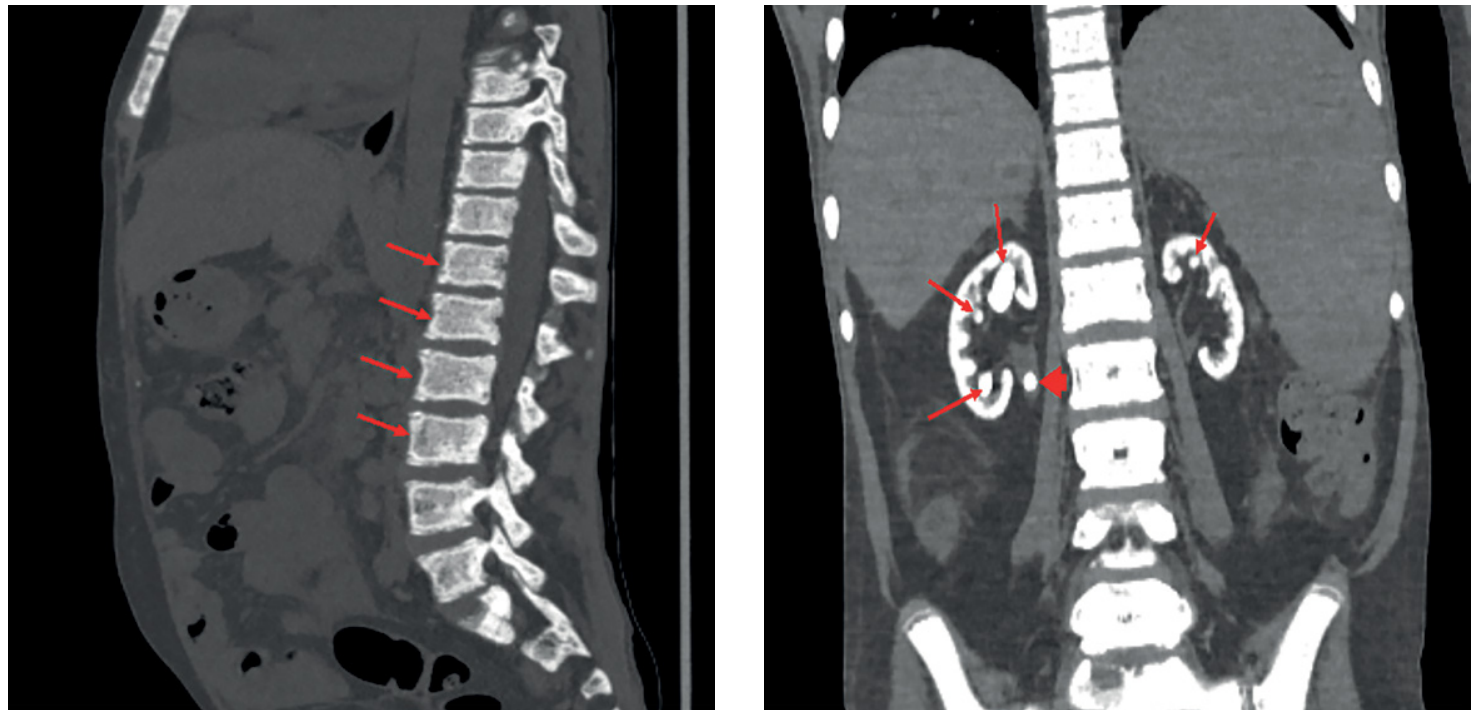

Рис. 2. Мультиспиральная компьютерная томография грудного и поясничного отделов позвоночника.

Описание. Диффузный остеосклероз пояснично-крестцового отдела позвоночника, неравномерное утолщение кортикального слоя (стрелки). Нефрокальциноз, конкременты в чашечках почек (стрелки), камень в лоханке (головка стрелки).
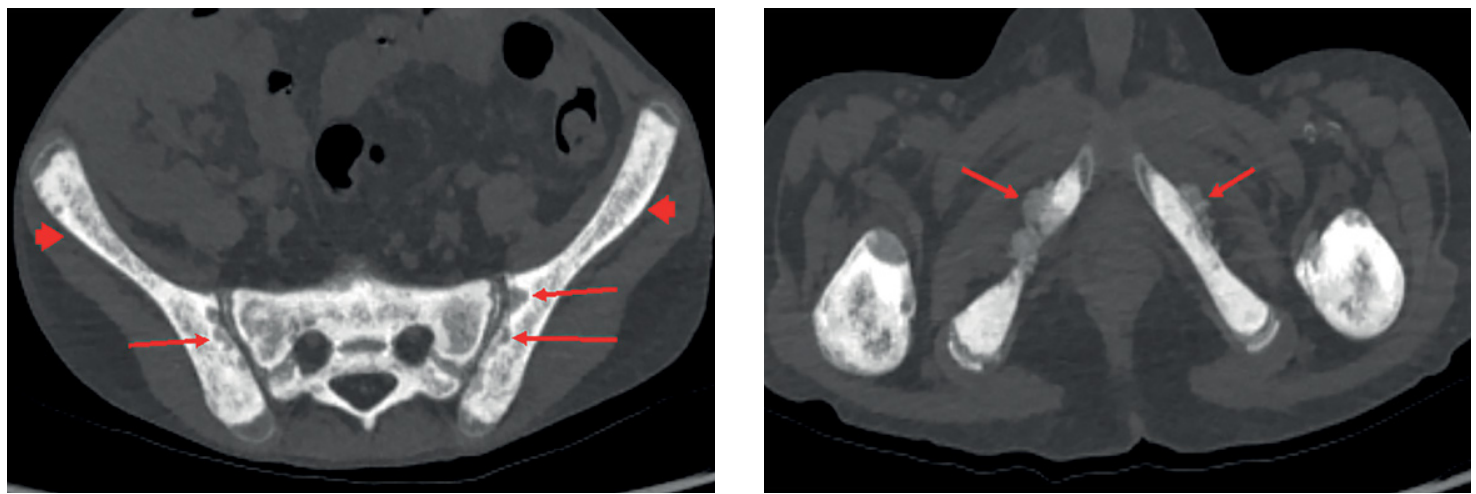

Рис. 3. Мультиспиральная компьютерная томография костей таза.

Описание. Диффузный остеосклероз костей таза с участками разряжения костной ткани в субхондральных отделах (стрелки), неравномерное утолщение кортикального слоя (головки стрелок). «Облаковидные» периостальные разрастания лобковых костей (стрелки) на фоне диффузного остеосклероза.

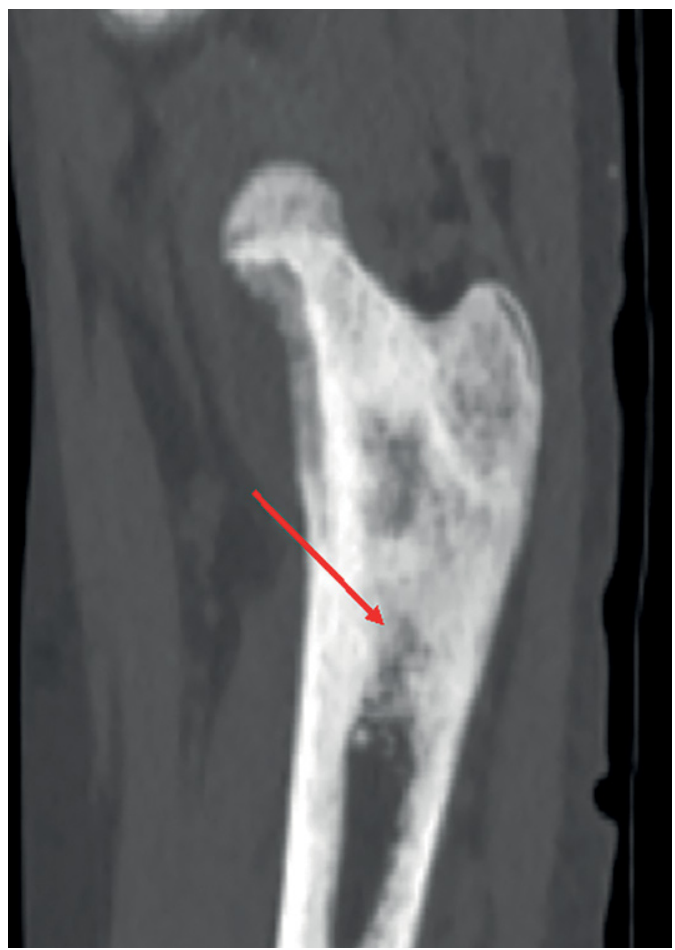

Рис. 4. Мультиспиральная компьютерная томография тазобедренных суставов.

Описание: Диффузный остеосклероз бедренной кости с распространением в костномозговой канал (стрелка). 
отмечена полная нормализация биохимических показателей фосфорно-кальциевого обмена - кальций общий 2,32 ммоль/л, фосфор 1,03 ммоль/л (табл.1). Однако через 2 мес после инъекции деносумаба вновь усилились боли в костях, особенно в области седалищного бугра справа, наблюдалось повышение уровня кальция и фосфора в крови, через 3 мес - значительное повышение маркеров костного метаболизма при стабильных показателях гипопротеинемии, гипоальбуминемии, СКФ, печеночных ферментов, отсутствии анемии и прогрессирования лейкопении и тромбоцитопении (табл. 1). Через 3,5 мес после первой инъекции пациенту было рекомендовано сделать повторную инъекцию деносумаба в дозе 60 мг с дальнейшей частотой введения в зависимости от клинико-лабораторных показателей. Ввиду отсутствия официальных показаний в инструкции к препарату для лечения оксалатной остеопатии, деносумаб был назначен после проведения врачебной комиссии.

При повторной госпитализации через 6 мес при детальном опросе пациента и его матери выяснено, что боли в костях таза уменьшаются после инъекции деносумаба на 2-3-й день и пациент не нуждается в приеме обезболивающих препаратов около 1,5 мес. По данным лабораторных исследований через 2 мес после последней инъекции деносумаба вновь определялись гиперкальциемия и значительное повышение маркера костной резорбции $\beta$-CrossLaps (табл. 1). На 2-е сутки после инъекции деносумаба отмечалось снижение уровня кальция, скорректированного на альбумин, с 3,32 ммоль/л до 2,44 ммоль/л, $\beta$-CrossLaps - с 6 нг/мл до 0,68 нг/мл. При выписке из стационара пациенту были даны рекомендации о необходимости выполнения инъекций деносумаба в дозе 60 мг 1 раз в 45-60 дней.

\section{ОБСУЖДЕНИЕ}

Гипероксалурия вследствие высокой экскреции оксалата с мочой прежде всего сопровождается поражением почек, которое может проявиться в любом возрасте, но чаще всего клинические симптомы возникают в раннем детском возрасте - до 50\% случаев у детей в возрасте до 5 лет и примерно в 10\% случаев на первом году жизни [18]. Спектр заболеваний варьирует от мягких форм мочекаменной болезни до тяжелых форм нефролитиаза и нефрокальциноза, приводящих к терминальной стадии ХБП. Выраженность клинических проявлений во многом зависит от степени нарушения обмена глиоксилата, наличия нефролитиаза и обструкции мочевыводящих путей [19].

В структуре различных причин ХБП у детей первичная гипероксалурия типа 1, по данным национальных регистров США, Европы, Японии, составляет менее 2\% [12]. Примерно в 30\% случаев диагноз устанавливается уже на терминальной стадии ХПН, у некоторых пациентов гипероксалурия выявляется только после рецидива нефрокальциноза аллотрансплантата почки [20, 21]. Прогрессирующее снижение СКФ приводит к возрастанию уровня оксалатов в плазме и осаждению его в различных органах и тканях, прежде всего в тканях, богатых кровеносными сосудами, с развитием в них воспалительной реакции. Следствием поражения костного мозга служит развитие вторичной анемии, резистентной к лечению эритропоэтином, в некоторых случаях может развиваться панцитопения [22, 23].

Отложение кристаллов оксалата кальция в костной ткани сопровождается болями в костях, патологическими переломами преимущественно шейки бедренной кости, деформациями костей, в тяжелых случаях формируются субпериостальные тофусы [24, 25]. Оксалатная остеопатия, как правило, представляет собой симметричное поражение скелета с преимущественным вовлечением метафизов. Типичные рентгенологические признаки включают плотные метафизарные полосы, расположенные у края метафизов, склероз прилегающих диафизов, утолщение кортикального слоя, кистозные изменения костей, увеличение плотности костной ткани в позвонках и гребнях подвздошных костей $[19,25,26]$.

Отложение оксалатных депозитов в сердечно-сосудистой системе может обуславливать развитие гипертрофической кардиомиопатии, сердечной недостаточности, различных нарушений сердечного ритма и проводимости, микроциркуляторные нарушения в результате повреждения сосудистой стенки с развитием эндотелиальной дисфункции [27]. Описаны случаи ишемических инсультов, вызванных нарушением микроциркуляции и эмболией кристаллами оксалата кальция $[28,29]$.

Эндокринные заболевания являются достаточно частой патологией у пациентов с первичной гипероксалурией. В качестве осложнения системного оксалоза со стороны эндокринной системы описано развитие ПТГ-независимой гиперкальциемии, первичного гипотиреоза, первичного гипогонадизма [7].

Первичная гипероксалурия - это трудно поддающееся лечению заболевание. Знание особенностей клинических проявлений, своевременная диагностика и начало лечения до развития ХБП имеют важное прогностическое значение для пациентов. На ранних стадиях лечение преимущественно направлено на сохранение функции почек, предупреждение развития нефролитиаза и нефрокальциноза. Независимо от типа гипероксалурии важно соблюдение адекватного питьевого режима с потреблением жидкости в объеме 2-3 л в сутки для снижения концентрации мочи и уменьшения склонности к образованию оксалатно-кальциевых камней [30]. При определенных мутациях в гене AGXT (c.508G >A (p.Gly170Arg), c.454T>A (p.Phe152lle), c.731T>C (p.lle244Thr)) доказан положительный эффект от использования высоких доз пиридоксина (витамин В) [31]. Снижение экскреции оксалатов с мочой на $30 \%$ и более от исходного уровня свидетельствует об эффективности лечения [32]. В некоторых случаях терапия пиридоксином в сочетании с повышенной гидратацией приводит к нормализации экскреции оксалатов и может оказать влияние на исход трансплантации, в том числе значительно улучшить выживаемость почечного трансплантата [33]. Однако любая консервативная терапия первичной гипероксалурии является паллиативной и позволяет лишь замедлить прогрессирование заболевания, но не предотвратить его полностью. Изолированная трансплантация печени целесообразна только на ранних стадиях заболевания при относительно сохранной функции почек (СКФ 50-70 мл/мин/1,73 м²). Наиболее оправданным методом 
Таблица 4. Результаты исследования 25(OH)D и других метаболитов витамина D методом высокоэффективной жидкостной хроматографии с тандемным масс-спектрометрическим детектированием (ВЭЖХ-МС/MC)

\begin{tabular}{lcc}
\hline \multicolumn{1}{c}{ Параметр } & Значение & Референсный интервал \\
\hline $25(\mathrm{OH}) \mathrm{D}_{2^{\prime}} \mathrm{Hг} / \mathrm{Mл}$ & $<0,1$ & $20-60$ \\
$25(\mathrm{OH}) \mathrm{D}_{3^{\prime}} \mathrm{Hг} / \mathrm{Mл}$ & 23,6 & $20-60$ \\
$25(\mathrm{OH}) \mathrm{D}, \mathrm{Hг} / \mathrm{Mл}$ & 23,6 & $20-60$ \\
$24,25(\mathrm{OH})_{2} \mathrm{D}_{3^{\prime}} \mathrm{Hr} / \mathrm{M} \pi$ & 0,6 & $0,5-5,6$ \\
$3-\mathrm{epi}-25(\mathrm{OH}) \mathrm{D}_{3^{\prime}} \mathrm{Hг} / \mathrm{M} \pi$ & 1,1 & $1-10$ \\
Соотношение $25(\mathrm{OH}) \mathrm{D}_{3} / 24,25(\mathrm{OH})_{2} \mathrm{D}_{3}$ & 39,3 & $7-25$ \\
$1,25(\mathrm{OH})_{2} \mathrm{D}_{3^{\prime}}$ Пг/мл & 29 & $18-64$ \\
\hline
\end{tabular}

лечения первичной гипероксалурии типа 1 является комбинированная трансплантация печени и почки [26].

Представленный клинический случай наглядно демонстрирует тяжесть течения обсуждаемого редкого генетического заболевания, подчеркивая необходимость своевременной диагностики и коррекции нарушений. Диагностика причины нефролитиаза, нефрокальциноза произошла на поздней стадии ХБП, когда пациент уже несколько лет находился на заместительной почечной терапии и развилось отложение оксалатов в различных органах и тканях. На рис. 2 отчетливо видны массивные отложения оксалатов в паренхиме почек, визуализирующиеся как конгломераты извести. Выраженная панцитопения у нашего пациента, вероятнее всего, обусловлена склеротическими и фиброзными изменениями костного мозга вследствие прогрессирования остеосклероза с сужением костномозговых полостей (рис. 4) на фоне основного заболевания в сочетании с проводимой иммуносупрессивной терапией. Выраженные боли в костях, особенно в тазовых, связаны с отложением кристаллов оксалата кальция в костной ткани, образованием субпериостальных тофусов, прогрессирующим остеосклерозом.

Необходимо отметить, что в возрасте 20 лет наш пациент перенес ОНМК по ишемическому типу в бассейне левой средней мозговой артерии с развитием легкого правостороннего центрального гемипареза, что описано у больных первичной гипероксалурией и может быть обусловлено нарушением микроциркуляции и эмболией кристаллами оксалата кальция [28, 29].

Особенностью представленного клинического случая является позднее развитие ПТГ-независимой гиперкальциемии с признаками высокого костного обмена. Этиология ПТГ-независимой гиперкальциемии при первичной гипероксалурии типа 1 многофакторная. Отложения оксалата кальция напрямую индуцируют деструкцию костной ткани посредством резорбции кости, что было доказано в ряде исследований биоптатов кости. Дополнительно происходит дифференцировка макрофагов в участках хронического воспаления в ответ на инородные тела (депозиты оксалатов кальция). Реагируя на местные стимулы, макрофаги эволюционируют от мононуклеарных клеток, поглощающих чужеродный материал, в многоядерные гигантские клетки с последующей резорбцией более крупных субстратов, включая костную ткань. Предполагается, что эти многоядерные клетки, сливаясь, образуют гигантоклеточные гранулемы, секретирующие различные цитокины, включая интерлейкин-1, стимулирующие активность остеокластов [7]. Возмож- но, в дополнение к прямой резорбтивной способности активированные в гранулемах макрофаги обладают активностью 1а-гидроксилазы (СРP27B1), ответственной за гидроксилирование 25(OH)D в 1,25(OH) $\mathrm{D}_{3}$ (кальцитриол), что приводит к увеличению абсорбции кальция из кишечника и высвобождению кальция из костей [7]. Однако у нашего пациента при анализе метаболитов витамина D и соотношения концентрации различных метаболитов значимых отклонений выявлено не было, что позволяет исключить выше указанный механизм и подтвердить превалирование механизма высокой костной резорбции (табл. 4).

Повышение маркеров костного ремоделирования, особенно маркера костной резорбции, с одной стороны, может быть обусловлено длительно существующей ХБП [34]. Однако подобное превышение в 4-6 раз при низком уровне ПТГ, как в представленном случае, трудно объяснить только вкладом ХБП. В этой ситуации больше играет роль локальная костная резорбция, обусловленная вышеописанными процессами, вследствие отложения оксалатных депозитов.

Кроме того, наш пациент в возрасте 17 лет перенес удаление 15 зубов, а также экзостозов верхней и нижней челюсти. Подобные явления встречаются довольно редко, однако в литературе имеются описания 12 отдельных клинических случаев отложения кристаллов оксалата кальция в пульпе зубов, альвеолярных отростках, резорбции челюстных костей, развития ортодонтических нарушений, таких как гиподонтия, микродонтия и периодонтит, у пациентов с первичной гипероксалурией [35]. Резорбция зубов при данном заболевании объясняется хроническим воспалением, а также присутствием остеокласт-подобных клеток, окружающих депозиты кристаллов оксалата кальция [24].

Среди эндокринных расстройств у пациента развились гипергонадотропный гипогонадизм вследствие отложения кристаллов оксалата кальция в яичках и, возможно, длительной терапии глюкокортикоидными гормонами в рамках супрессивной терапии после трансплантации печени и почки, а также субклинический гипотиреоз, что характерно для больных с первичной гипероксалурией типа 1 и согласуется с литературными данными.

Учитывая наличие выраженных болей в костях, гиперкальциемии, высоких показателей костного метаболизма, была инициирована терапия деносумабом. Назначение деносумаба позволило временно достичь нормализации показателей фосфорно-кальциевого обмена, существенно снизить активность костной резорбции, улучшить 
клинические показатели, однако через 2 мес после первой инъекции отмечено повышение уровня кальция и фосфора, а через 3,5 мес - возвращение почти к исходным значениям. По данным лабораторных исследований через 2 мес после второй инъекции деносумаба на фоне низкого уровня ПТГ вновь отмечалось повышение маркеров костного метаболизма, что свидетельствует о более коротком антирезорбтивном действии деносумаба при первичной гипероксалурии типа 1 и необходимости более частого его введения, чем позиционируется для лечения остеопороза. К настоящему времени нам удалось найти одну работу по эффективному применению деносумаба в течение 18 мес для лечения гиперкальциемии и оксалатной остеопатии, подтвержденной гистологически, при первичной гипероксалурии типа 1 у женщины 69 лет после аллотрансплантации печени и почки с развитием третичного гиперпаратиреоза [36].

В литературе имеются единичные сообщения о применении памидроната для лечения гиперкальциемии при первичной гипероксалурии типа 1, однако эффект также был временным и сохранялся в течение 3 мес [37]. В нашем случае назначение бисфосфонатов противопоказано вследствие выраженной ХБП.

Представленное клиническое наблюдение является первым, где пациенту с тяжелой остеопатией и ПтГнезависимой гиперкальциемией на фоне первичной гипероксалурии инициирована терапия деносумабом с положительным клиническим эффектом.

\section{ЗАКЛЮЧЕНИЕ}

Представлено первое описание клинического случая лечения молодого пациента с генетически подтверж- денным врожденным нарушением обмена оксалатов (первичная гипероксалурия типа 1), подвергшегося трансплантации печени и почки через 10 лет от начала заболевания, уже на терминальной стадии ХБП. Осложнениями основного заболевания, резко снижающими качество жизни, являлись тяжелая остеопатия и ПТГ-независимая гиперкальциемия, для коррекции которых был назначен деносумаб - моноклональное человеческое антитело к лиганду рецептора активатора ядерного фактора каппа бета (RANKL). Назначение деносумаба в дозе 60 мг каждые 2 мес позволило скорректировать гиперкальциемию и уменьшить оссалгии. Данный клинический случай демонстрирует важность исключения генетически обусловленного дефекта синтеза печеночных ферментов как причины рецидивирующего нефролитиаза и нефрокальциноза, приводящего к широкому спектру метаболических, костных и эндокринных нарушений. Своевременная диагностика и лечение первичной гипероксалурии являются критически важными для увеличения продолжительности и улучшения качества жизни пациентов.

\section{ДОПОЛНИТЕЛЬНАЯ ИНФОРМАЦИЯ}

Согласие пациента. Пациент добровольно подписал информированное согласие на публикацию персональной медицинской информации в обезличенной форме в журнале «Остеопороз и остеопатии».

Участие авторов. Все авторы внесли значимый вклад в проведение поисково-аналитической работы и подготовку статьи, прочли и одобрили финальную версию до публикации.

Конфликт интересов. Авторы заявляют об отсутствии конфликта интересов, связанных с публикацией настоящей статьи.

\section{СПИСОК ЛИТЕРАТУРЫ | REFERENCES}

1. Sas DJ, Harris PC, Milliner DS. Recent advances in the identification and management of inherited hyperoxalurias. Urolithiasis. 2019:47(1):79-89. doi: 10.1007/s00240-018-1093-3.

2. Zhang X., Roe SM., Pearl LH., Danpure CJ. Crystallization and preliminary crystallographic analysis of human alanine:glyoxylate aminotransferase and its polymorphic variants. Acta Crystallogr D Biol Crystallogr. 2001;57(Pt 12):1936-1937. doi: $10.1107 /$ s0907444901017334.

3. Cochat $P$, Hulton S-A, Acquaviva $C$, et al. Primary hyperoxaluria Type 1: indications for screening and guidance for diagnosis and treatment. Nephrol Dial Transplant. 2012;27(5):1729-36. doi: 10.1093/ndt/gfs078.

4. Филиппова Т.В., Светличная Д.В., Руденко В.И., и др. Генетические аспекты первичной гипероксалурии: эпидемиология, этиология, патогенез и клинические проявления заболевания // Урология. - 2019. - №6. - C.125-130. [Filippova TV, Svetlichnaya DV, Rudenko VI, et al. Genetic aspects of primary hyperoxaluria: epidemiology, ethiology, pathogenesis, and clinical signs of the disorder. Urologiia. 2019;(6):125-130. (In Russ.)]. doi: 10.18565/urology.2019.6.125-130.

5. Williams EL, Acquaviva C, Amoroso A, et al. Primary hyperoxaluria type 1: update and additional mutation analysis of the AGXT gene. Hum Mutat. 2009;30(6):910-917. doi: 10.1002/humu.21021.

6. Cochat P, Rumsby G. Primary Hyperoxaluria. N Engl J Med. 2013;369(7):649-658. doi: 10.1056/NEJMra1301564.

7. Murad S, Eisenberg Y. Endocrine manifestations of primary hyperoxaluria. Endocrine Practice. 2017;23(12):1414-1424 doi: 10.4158/EP-2017-0029.

8. Grenda R., Kaliciński P. Combined and sequential liver-kidney transplantation in children. Pediatr Nephrol 2018;33(12):2227-2237. doi: 10.1007/s00467-017-3880-4
9. Kotb MA, Hamza AF, Abd El Kader H, et al. Combined liver-kidney transplantation for primary hyperoxaluria type I in children: Single Center Experience. Pediatr Transplant. 2019;23(1):e13313. doi: 10.1111/petr.13313.

10. Simpson N, Cho YW, Cicciarelli JC, et al. Comparison of renal allograft outcomes in combined liver-kidney transplantation versus subsequent kidney transplantation in liver transplant recipients: Analysis of UNOS Database. Transplantation. 2006;82(10):1298-1303. doi: 10.1097/01.tp.0000241104.58576.e6.

11. Calinescu AM, Wildhaber BE, Poncet A, et al. Outcomes of Combined Liver-Kidney Transplantation in Children: Analysis of the Scientific Registry of Transplant Recipients. American Journal of Transplantation. 2014;14(12):2861-2868. doi: 10.1111/ajt.12935

12. Harambat J, van Stralen KJ, Espinosa L, et al. Characteristics and Outcomes of Children with Primary Oxalosis Requiring Renal Replacement Therapy. Clin J Am Soc Nephrol. 2012;7(3):458-465. doi: 10.2215/CJN.07430711.

13. Konkolová J, Chandoga J, Kováčik J, et al. Severe child form of primary hyperoxaluria type 2 - a case report revealing consequence of GRHPR deficiency on metabolism. BMC Med Genet 2017;18. doi: 10.1186/s12881-017-0421-8.

14. Fu Y, Rope R, Fargue $S$, et al. A mutation creating a highly active out-of-frame alternative translation initiation site within the $5^{\prime}$ UTR of the GRHPR gene causing primary hyperoxaluria type II. Clin Genet. 2015:88(5):494-498. doi: 10.1111/cge.12541.

15. Cregeen DP, Williams EL, Hulton S, Rumsby G. Molecular analysis of the glyoxylate reductase (GRHPR) gene and description of mutations underlying primary hyperoxaluria type 2. Hum Mutat. 2003;22(6):497. doi: 10.1002/humu.9200.

16. Dindo M, Conter C, Oppici E, et al. Molecular basis of primary hyperoxaluria: clues to innovative treatments. Urolithiasis. 2019:47(1):67-78. doi: 10.1007/s00240-018-1089-z. 
17. Fang $X, \mathrm{He} L, X u G$, et al. Nine novel HOGA1 gene mutations identified in primary hyperoxaluria type 3 and distinct clinical and biochemical characteristics in Chinese children. Pediatr Nephrol. 2019;34(10):1785-1790. doi: 10.1007/s00467-019-04279-7.

18. Abukhatwah MW, Almalki SH, Althobaiti M., et al. Primary hyperoxaluria Type 1. Medicine (Baltimore). 2020;99(25). doi: 10.1097/MD.0000000000020371

19. Rootman MS, Mozer-Glassberg Y, Gurevich M, et al. Imaging features of primary hyperoxaluria. Clinical Imaging. 2018;52:370-376. doi: 10.1016/j.clinimag.2018.09.009.

20. Rios JFN, Zuluaga M, Higuita LMS, et al. Primary hiperoxaluria diagnosed after kidney transplantation: report of 2 cases and literature review. Brazilian Journal of Nephrology. 2017;39(4):462-466. doi: 10.5935/0101-2800.20170081.

21. Lorenzo V, Torres A, Salido E. Primary hyperoxaluria. Nefrologia 2014;34(3):398-412. doi: 10.3265/Nefrologia.pre2014.Jan.12335.

22. Mykytiv V, Campoy Garcia F. Anemia in patient with primary hyperoxaluria and bone marrow involvement by oxalate crystals. Hematol Oncol Stem Cell Ther. 2018;11(2):118-121. doi: 10.1016/j.hemonc.2017.07.007

23. Sharma S, Rao RN, Pani KC, Paul P. Bone marrow oxalosis: An unusual cause of cytopenia in end-stage renal disease; report of two cases. Indian J Pathol Microbiol. 2018;61(2):268-270. doi: 10.4103/IJPM.IJPM_572_17.

24. Bacchetta J, Boivin G, Cochat P. Bone impairment in primary hyperoxaluria: a review. Pediatr Nephrol. 2016;31(1):1-6. doi: 10.1007/s00467-015-3048-z.

25. El Hage S., Ghanem I., Baradhi A., et al. Skeletal features of primary hyperoxaluria type 1, revisited. J Child Orthop 2008;2(3):205-210. doi: 10.1007/s11832-008-0082-4.

26. Strauss SB, Waltuch T, Bivin W, et al. Primary hyperoxaluria: spectrum of clinical and imaging findings. Pediatr Radiol. 2017;47(1):96-103. doi: 10.1007/s00247-016-3723-7.
27. Mookadam F, Smith T, Jiamsripong $P$, et al. Cardiac abnormalities in primary hyperoxaluria. Circ J. 2010;74(11):2403-2409. doi: 10.1253/circj.cj-10-0107.

28. Rao NM, Yallapragada A, Winden KD, et al. Stroke in Primary Hyperoxaluria Type I. J Neuroimaging. 2014; 4(4):411-413. doi: 10.1111/jon.12020

29. Lammie GA, Wardlaw J, Dennis M. Thrombo-embolic stroke, moya-moya phenomenon and primary oxalosis. Cerebrovasc Dis. 1998:8(1):45-50. doi: 10.1159/000015815.

30. Hoppe B. An update on primary hyperoxaluria. Nat Rev Nephrol 2012:8(8):467-475. doi: 10.1038/nrneph.2012.113.

31. Monico CG, Rossetti S, Olson JB, Milliner DS. Pyridoxine effect in type I primary hyperoxaluria is associated with the most common mutant allele. Kidney Int. 2005;67(5):1704-1709. doi: 10.1111/j.1523-1755.2005.00267.x.

32. Hoyer-Kuhn H, Kohbrok S, Volland R, et al. Vitamin B6 in Primary Hyperoxaluria I: First Prospective Trial after 40 Years of Practice. Clin J Am Soc Nephrol. 2014;9(3):468-477. doi: 10.2215/CJN.06820613.

33. Lorenz EC, Lieske JC, Seide BM, et al. Sustained pyridoxine response in primary hyperoxaluria type 1 recipients of kidney alone transplant. Am J Transplant. 2014;14(6):1433-1438. doi: 10.1111/ajt.12706.

34. Chiang $C$. The use of bone turnover markers in chronic kidney disease-mineral and bone disorders. Nephrology (Carlton). 2017;22 Suppl 2:11-13. doi: 10.1111/nep.13014.

35. Cazzolla AP, Zhurakivska K, Ciavarella D, et al. Primary hyperoxaluria: Orthodontic management in a pediatric patient: A case report. Spec Care Dentist. 2018;38(4):259-265. doi: 10.1111/scd.12302

36. Somme F, Blondet C, Matuszak J, Heimburger C. Evaluation of Oxalate Osteopathy Secondary to Hyperoxaluria With 18F-FDG PET/CT and 99mTc-HMDP Bone Scan. Clin Nucl Med. 2019;44(2):123-124. doi:10.1097/RLU.0000000000002386

37. Yamaguchi K., Grant J., Noble-Jamieson G., et al. Hypercalcaemia in primary oxalosis: role of increased bone resorption and effects of treatment with pamidronate. Bone 1995;16(1):61-67

\section{ИНФОРМАЦИЯ ОБ АВТОРАХ [AUTHORS INFO]}

*Рожинская Людмила Яковлевна, д.М.Н., профессор [Liudmila Ya. Rozhinskaya, MD, PhD, Professor]; адрес: Россия, 117036, Москва, ул. Дм. Ульянова, д. 11 [address: 11 Dm. Ulyanova street, 117036 Moscow, Russia]; ORCID: http://orcid.org/0000-0001-7041-0732; eLibrary SPIN: 5691-7775; e-mail: Irozhinskaya@gmail.com

Голоунина Ольга Олеговна, студент [Olga O. Golounina, Student, Medical faculty];

ORCID: https://orcid.org/0000-0003-2320-1051; eLibrary SPIN: 7793-2123; e-mail: olga.golounina@mail.ru

Гребенникова Татьяна Алексеевна, К.M.H. [Tatiana A. Grebennikova, MD, PhD]; ORCID: http://orcid.org/0000-0003-1413-1549; eLibrary SPIN: 4380-5447; email: grebennikova@hotmail.com

Ковалевич Лилия Дмитриевна [Liliya D. Kovalevich]; ORCID: https://orcid.org/0000-0001-8958-8223; eLibrary SPIN: 1642-5694; email: liliyakovalevich@gmail.com

Гронская Софья Александровна, аспирант [Sofya A. Gronskaia, resident]; ORCID: http://orcid.org/0000-0001-7055-2407; eLibrary SPIN: 7624-0391; email: sofyaants@gmail.com

Богданов Виктор Павлович [Victor P. Bogdanov]; ORCID: http://orcid.org/0000-0001-6377-9056; eLibrary SPIN: 9956-8495; email: siberman@yandex.ru

Белая Жанна Евгеньевна, д.м.н., профессор [Zhanna E. Belaya, MD, PhD, Professor];

ORCID: http://orcid.org/0000-0002-6674-6441; eLibrary SPIN: 4746-7173; email: jannabelaya@gmail.com

\section{ИНФОРМАЦИЯ}

Рукопись получена: 04.12.2020. Одобрена к публикации: 16.01.2021.

\section{ЦИТИРОВАТЬ:}

Рожинская Л.Я., Голоунина О.О., Гребенникова Т.А., Ковалевич Л.Д., Гронская С.А., Богданов В.П., Белая Ж.Е. Применение деносумаба для лечения гиперкальциемии и гипероксалатной остеопатии у пациента с первичной гипероксалурией типа 1: описание клинического случая и обзор литературы // Остеопороз и остеопатии. - 2020. - Т. 23. - №3. C.XXX-XXX. doi: https://doi.org/10.14341/osteo12693

\section{TO CITE THIS ARTICLE:}

Rozhinskaya LYa, Golounina OO, GrebennikovaTA, Kovalevich LD, Gronskaya SA, Bogdanov VP, Belaya ZE. Clinical experience of using denosumab in the treatment of hypercalcemia and oxalate osteopathy for a young patient with primary hyperoxaluria type 1. Osteoporosis and bone diseases. 2020;23(3):XXX-XXX. doi: https://doi.org/10.14341/osteo12693 